\title{
BioéthiqueOnline
}

\section{Adolescents, neurosciences et prise de décisions médicales : devrions-nous revoir certaines dispositions du Code civil du Québec?}

\section{Brigitte Brabant}

Volume 5, 2016

URI : https://id.erudit.org/iderudit/1044262ar

DOI : https://doi.org/10.7202/1044262ar

Aller au sommaire du numéro

Éditeur(s)

BioéthiqueOnline

ISSN

1923-2799 (numérique)

Découvrir la revue

Citer cet article

Brabant, B. (2016). Adolescents, neurosciences et prise de décisions médicales : devrions-nous revoir certaines dispositions du Code civil du Québec? BioéthiqueOnline, 5. https://doi.org/10.7202/1044262ar
Résumé de l'article

Au Québec, le mineur de 14 ans et plus peut consentir seul aux soins médicaux requis par son état de santé, sauf exception. Or, les données provenant des neurosciences indiquent que : 1) l'acquisition de la " maturité cérébrale » est un processus continu, pouvant durer jusqu'à la mi-vingtaine, et que 2) certaines habiletés décisionnelles de l'adolescent, comme l'évaluation des risques et bénéfices à court et à long terme, seraient différentes de celles des adultes, surtout dans les situations émotivement chargées. Nous soumettons que, dans une perspective de protection de l'intérêt supérieur de l'enfant, la réalité neurophysiologique de l'adolescent devrait être prise en compte en contexte de soins de santé. Ainsi, nous questionnons le caractère adéquat de la présomption de capacité d'exercer un jugement de façon autonome dès l'âge de 14 ans et soulignons l'aspect paradoxal de la possibilité d'une intervention judiciaire en cas de refus de traitement. Nous questionnons également la pertinence de considérations d'âge dans le cadre de la relation entre le médecin et son patient, dès lors que la détermination de la capacité décisionnelle est une composante essentielle de l'obtention d'un consentemen libre et éclairé. Enfin, nous questionnons l'exclusion des parents du processus décisionnel, à la fois en regard de leur responsabilité parentale et des bénéfices que peuvent représenter leurs conseils pour l'adolescent. Par conséquent, nous soumettons qu'une évaluation du bien-fondé de l'octroi d'un pouvoir décisionnel à l'adolescent de 14 ans et plus serait souhaitable et qu'un régime uniformisé tout au long de la minorité, avec de possibles exceptions, reflèterait mieux les connaissances scientifiques actuelles.
Ce document est protégé par la loi sur le droit d'auteur. L'utilisation des services d’Érudit (y compris la reproduction) est assujettie à sa politique d'utilisation que vous pouvez consulter en ligne.

https://apropos.erudit.org/fr/usagers/politique-dutilisation/ 


\title{
Adolescents, neurosciences et prise de décisions médicales: devrions-nous revoir certaines dispositions du Code civil du Québec?
}

\author{
ARTICLE (RÉVISION PAR LES PAIRS / PEER-REVIEWED) \\ Brigitte Brabant ${ }^{1}$ \\ Reçu/Received: 10 Aug 2015 \\ Publié/Published: 3 Feb 2016 \\ Éditeurs/Editors: Vanessa Chenel \& Jean Poupart \\ Évaluateurs externes/Peer-Reviewers: Emmanuelle Lévesque \& Emmanuelle Marceau
}

2016 B Brabant, Creative Commons Attribution 4.0 International License

\section{Résumé}

Au Québec, le mineur de 14 ans et plus peut consentir seul aux soins médicaux requis par son état de santé, sauf exception. Or, les données provenant des neurosciences indiquent que : 1) l'acquisition de la " maturité cérébrale » est un processus continu, pouvant durer jusqu'à la mivingtaine, et que 2) certaines habiletés décisionnelles de l'adolescent, comme l'évaluation des risques et bénéfices à court et à long terme, seraient différentes de celles des adultes, surtout dans les situations émotivement chargées. Nous soumettons que, dans une perspective de protection de l'intérêt supérieur de l'enfant, la réalité neurophysiologique de l'adolescent devrait être prise en compte en contexte de soins de santé. Ainsi, nous questionnons le caractère adéquat de la présomption de capacité d'exercer un jugement de façon autonome dès l'âge de 14 ans et soulignons l'aspect paradoxal de la possibilité d'une intervention judiciaire en cas de refus de traitement. Nous questionnons également la pertinence de considérations d'âge dans le cadre de la relation entre le médecin et son patient, dès lors que la détermination de la capacité décisionnelle est une composante essentielle de l'obtention d'un consentement libre et éclairé. Enfin, nous questionnons l'exclusion des parents du processus décisionnel, à la fois en regard de leur responsabilité parentale et des bénéfices que peuvent représenter leurs conseils pour l'adolescent. Par conséquent, nous soumettons qu'une évaluation du bien-fondé de l'octroi d'un pouvoir décisionnel à l'adolescent de 14 ans et plus serait souhaitable et qu'un régime uniformisé tout au long de la minorité, avec de possibles exceptions, reflèterait mieux les connaissances scientifiques actuelles.

\section{Mots clés}

neurosciences, cerveau de l'adolescent, consentement aux soins, maturité, critère d'âge

\section{Abstract}

In the Province of Quebec, an adolescent aged 14 years or older may, subject to a few exceptions, consent alone to medical treatment required for his or her condition. However, neuroscience research on the adolescent's brain indicates that: 1) the acquisition of "brain maturity" is a continuing process, which can last until the mid-twenties and 2) certain decisional capacities such as assessment of short and long-term risks and benefits differ from those of adults, particularly in emotionally charged situations. I argue that, from the perspective of a child's best interest, the neurophysiological capacity of the adolescent should be taken into consideration in the health care context. Thus, I question the adequacy of the presumption of ability to make autonomous judgments from the age of 14 , and point out the paradox of allowing judicial review of such judgments in cases of refusal of treatment. I also question the relevance of age considerations in the context of the doctor-patient relationship, where assessment of decisional ability is of the essence in obtaining an informed consent. Finally, I question the exclusion of parents from the decision-making process in light of both their continuing responsibility to protect their child's best interests and the benefits that parental guidance may provide to the adolescent. Consequently, I argue that a reconsideration of the case for giving decisional authority to the adolescent aged 14 or older is desirable and that a uniform standard, with a few possible exceptions, applicable throughout childhood would better reflect recent neuroscience findings.

\section{Keywords}

neurosciences, adolescent brain, consent to medical treatment, maturity, age-based criteria 


\section{Exonération}

Les évaluations des examinateurs externes sont prises en considération de façon sérieuse par les éditeurs et les auteurs dans la préparation des manuscrits pour publication. Toutefois, être nommé comme examinateur n'indique pas nécessairement l'approbation du manuscrit par cet examinateur. Les éditeurs de BioéthiqueOnline assument la responsabilité entière pour l'acceptation finale et la publication d'un article.

\section{Disclaimer}

Reviewer evaluations are given serious consideration by the editors and authors in the preparation of manuscripts for publication. Nonetheless, being named as a reviewer does not necessarily denote approval of a manuscript by the reviewer; the editors of BioéthiqueOnline take full responsibility for final acceptance and publication of an article.

Affiliations des auteurs / Author Affiliations

${ }^{1}$ Faculté de médecine, Université de Montréal, Québec, Canada

\section{Correspondance / Correspondence}

Brigitte Brabant, brigitte.brabant.1@umontreal.ca

\section{Remerciements}

Merci aux organisateurs et au jury de la Conférence de Montréal pour jeunes chercheurs en neuroéthique 2015 et aux éditeurs de BioéthiqueOnline pour leurs précieux commentaires et suggestions.

\section{Conflit d'intérêts}

Aucun déclaré

\section{Acknowledgements}

Thanks to the organizers and the jury of the 2015 Montreal Neuroethics Conference for Young Researchers and to the editors of BioéthiqueOnline for their valuable comments and suggestions.

\section{Conflicts of Interest}

None declared

\section{Introduction}

Au Québec, le mineur apte de 14 ans et plus peut consentir seul aux soins médicaux requis par son état de santé: hormis les cas d'urgence (lorsque sa vie est en danger ou son intégrité menacée) où le consentement du titulaire de l'autorité parentale ou du tuteur suffit, et pour le soumettre à des soins qu'il refuse, où l'autorisation du tribunal est nécessaire, le Code civil [1] lui attribue en ce domaine un pouvoir décisionnel autonome (art. 14, alinéa 2 et 16, alinéa 2). ${ }^{1}$ Comme régime d'application générale en matière de consentement aux soins, cette capacité juridique partielle établie en fonction de l'âge pour le mineur est unique au Canada [2,3]. Les conséquences de ce régime sont nombreuses et touchent à la fois l'adolescent, son entourage immédiat et les soignants. Parmi les plus importantes, mentionnons que la relation tripartite existant entre le médecin, les parents ${ }^{2}$ et l'enfant dans le domaine des soins pédiatriques se voit entièrement modifiée dès les 14 ans de l'enfant, puisque les parents sont alors en principe exclus du processus décisionnel et privés de l'accès à la plus grande partie des informations qui concernent leur enfant. ${ }^{3}$

Dans ce contexte, les récentes données provenant des neurosciences au sujet du cerveau des adolescents ont retenu notre attention. Nous avons voulu savoir si ces données scientifiques pouvaient jeter un éclairage nouveau sur ce régime particulier et si, le cas échéant, l'on pouvait questionner la pertinence de celui-ci d'un point de vue éthique. À cet égard, nous verrons que les découvertes portant sur le développement cérébral des adolescents alimentent, depuis déjà plusieurs années, des débats d'auteurs portant sur les règles applicables à ceux-ci en matière de consentement aux soins médicaux. Nous avons abordé la question du consentement aux soins du mineur de 14 ans et plus en regard des enjeux éthiques suivants : 1) quel est le poids pouvant être accordé aux données provenant des neurosciences quant à la capacité de jugement des adolescents en matière de prises de décisions médicales et 2) la place accordée à l'autonomie de l'adolescent de

\footnotetext{
${ }^{1}$ Nous nous limitons ici aux soins requis par l'état de santé ; les soins non requis par l'état de santé, l'aliénation d'une partie du corps, la recherche et le don d'organes faisant aussi l'objet de dispositions particulières pour le mineur.

${ }^{2}$ Pour des fins d'allégement du texte, nous utiliserons le terme « parents », ceux-ci étant titulaires de l'autorité parentale et tuteurs de plein droit (art. 192 et 597 et ss. C.c.Q).

${ }^{3}$ En cas d'hospitalisation, ce n'est qu'après 12 heures que les parents devront être informés, mais de l'hospitalisation seulement (art. 14, al. 2 C.c.Q). L'accès au dossier de l'enfant est toutefois permis : art. 21 Loi sur les services de santé et les services sociaux, L.R.Q. ch. S-4.2.
} 
14 ans et plus par notre Code civil devrait-elle être revue en conséquence de ces données, le tout en regard du critère du meilleur intérêt de l'enfant qui prévaut en matière de soins pédiatriques $[4,5]$ ?

En premier lieu, nous ferons un bref survol du cadre juridique applicable et de ses origines. Nous verrons ensuite quelles sont les découvertes des neurosciences qui pourraient être pertinentes à l'analyse de cette capacité décisionnelle et poursuivrons avec les enjeux éthiques soulevés plus haut. Nous étudierons ces enjeux de la perspective d'une capacité décisionnelle factuelle plutôt que juridique, tant au niveau individuel que collectif.

\section{Le cadre juridique et ses origines}

Au Québec, la capacité légale de l'adolescent de consentir seul aux soins en fonction de son âge a été établie au cours des années 1970. Les auteurs Kouri et Philips-Nootens [6] nous indiquent qu'à cette époque, et bien qu'aucune distinction n'ait été faite à cet égard dans la législation, l'on a voulu que l'accès à la contraception, au traitement des maladies à traitement obligatoire ou encore des cas d'abus d'alcool ou de drogues puisse être possible sans le consentement des parents et ce, de façon confidentielle. L'âge de 14 ans n'aurait été introduit dans la loi que par compromis politique, malgré les réticences alors exprimées devant l'Assemblée nationale par l'ancien ministre Camille Laurin, psychiatre. Ce seraient donc plutôt des considérations de santé publique, soit l'accès du mineur aux soins de santé, plutôt que la reconnaissance d'une capacité décisionnelle équivalente à celle d'un adulte, qui auraient mené à ce régime particulier. Le recours aux tribunaux, en cas de refus de traitement, et l'intervention des parents, en cas d'urgence, auraient été mis en place pour protéger l'adolescent contre l'erreur de jugement, contrairement au cas des adultes où le principe d'autonomie prévaut [6].

Ailleurs au Canada, la doctrine du « mineur mature » et diverses lois particulières portant sur le consentement aux soins ou encore sur la protection du bien-être de l'enfant, avec ou sans distinction fondée sur l'âge, régissent le pouvoir décisionnel du mineur en matière de soins médicaux [3]. Ainsi, d'abord pour une question d'accès à la contraception puis, de façon générale, l'on y a amené la notion de maturité dans la détermination du droit des adolescents à l'autonomie décisionnelle. La Cour Suprême du Canada, dans l'affaire A.C. c. Manitoba (Directeur des services à l'enfant et à la famille) [7] ("A.C. ») s'est penchée, en 2009, sur la portée de cette notion. Sans prétendre faire une analyse exhaustive de cette décision, nous en soulignerons les éléments qui paraissent essentiels à la compréhension de notre propos.

Tout d'abord, la Cour statuait que le critère de l'intérêt supérieur de l'enfant devait s'interpréter de façon à accorder au mineur qui démontrait suffisamment de maturité le droit de prendre sa décision de façon autonome, et donc de consentir seul aux soins ou de les refuser [7, par. 108]. La Cour soulignait également que cette maturité devait être évaluée au cas par cas, suivant une liste non exhaustive de facteurs, incluant la nature et les risques et bénéfices du traitement, la démonstration du discernement requis pour comprendre les renseignements qui permettraient à l'adolescent d'évaluer les conséquences de la décision ainsi que l'impact du contexte social, émotionnel ou médical. Cette évaluation devait mener à accorder de plus en plus de poids à l'opinion de l'adolescent selon son niveau de maturité, l'opinion d'un adolescent « mature » devant être respectée.

La Cour émettait cependant la réserve suivante [7, par. 95]:

« (...) dans les cas les plus graves où le refus de traitement présenterait un risque élevé de décès ou de déficience physique ou mentale permanente, il faudra nécessairement procéder à une évaluation minutieuse et approfondie de la maturité de l'adolescent pour déterminer si sa décision est prise de façon véritablement indépendante et s'il comprend et évalue bien la décision et ses conséquences possibles. " (nos soulignés) 
Il faut préciser que, dans cette affaire, la Cour se prononçait sur la constitutionnalité de la restriction du pouvoir décisionnel des adolescents de moins de 16 ans imposée par la loi manitobaine, statuant que cette restriction pouvait être acceptable dans la mesure où elle n'empêchait pas le mineur de faire la démonstration de sa maturité. Cette restriction entrait en jeu dans les cas de refus de soins à la fois du mineur et de ses parents, lorsque la vie ou la santé du mineur était en péril [7, par. 111]. Cette décision, bien que commentée par Kouri et Philips-Nootens [2,6], n'a pas traité de la législation québécoise.

Selon la recension des autorités qui est faite par la juge Abella dans l'affaire A.C., le régime réservé à l'adolescent en matière de consentement aux soins et ses origines sont similaires aux États-Unis, au Royaume-Uni et en Australie [7, par. 65-9]. En France, le Code de la santé publique [8] prévoit la recherche du consentement du mineur apte à exprimer sa volonté et à participer à la décision, sans égard à son âge. Tout comme le régime québécois, la question de la capacité légale de l'adolescent à consentir seul aux soins est donc encadrée, dans d'autres juridictions, par les principes d'autonomie et du meilleur intérêt de l'enfant. Son évaluation ne repose cependant pas clairement sur une réalité scientifique touchant le groupe des adolescents ou encore sur une définition de la capacité décisionnelle. De plus, le critère de " maturité » demeure imprécis quant aux paramètres applicables lors de l'examen "plus minutieux" dans les cas "les plus graves ». De façon similaire en droit québécois, aucune précision n'est donnée quant aux facteurs qui pourraient justifier le recours au tribunal dans les cas de refus de soins. Rappelons que la détermination de la maturité n'est pas requise dans notre régime québécois pour les adolescents de 14 ans et plus, puisque le seul facteur d'âge de 14 ans donne ouverture au pouvoir décisionnel de ceux-ci.

Nous verrons maintenant ce que les recherches portant sur l'étude du cerveau des adolescents, utilisant les techniques de neuroimagerie, peuvent nous apporter à cet égard.

\section{Les résultats de recherche en neurosciences}

En 2013, le psychologue Steinberg, auteur de plusieurs écrits qui concernent la période de l'adolescence, s'est intéressé au consentement aux soins médicaux des adolescents. Nous résumerons le bilan documenté qu'il faisait alors des résultats nous provenant des neurosciences [9]. D'entrée de jeu, Steinberg nous parle du fort consensus existant dans la communauté neuroscientifique quant à la nature des changements $s$, tant structurels que fonctionnels, qui surviennent dans le cerveau pendant la période de l'adolescence, sans uniformité dans le temps. II y aurait également consensus quant au fait que ces changements auraient lieu pendant une période beaucoup plus longue (jusqu'à la mi-vingtaine, pour certains) que ce que croyaient les chercheurs, incluant ceux provenant des sciences humaines, auparavant.

Selon lui, quatre changements structurels sont à noter:

1. La diminution de la matière grise dans les régions préfrontales durant la jeune adolescence, reflétant un élagage synaptique (processus pendant lequel les connexions inutilisées entre les neurones sont éliminées) créant un réseau d'analyse des informations plus efficace; cette période correspondrait à celle pendant laquelle on constate des améliorations majeures au niveau des habiletés cognitives et de raisonnement logique.

2. Autour de la puberté, une modification notable dans la distribution et la densité des récepteurs de la dopamine dans les voies qui relient le système limbique, où les émotions sont traitées et les punitions et récompenses expérimentées, au cortex préfrontal; cette activité élevée de la dopamine aurait une grande influence sur la recherche de sensations fortes. 
3. Une augmentation de la matière blanche dans le cortex préfrontal, qui serait due largement au processus de myélinisation, en vertu duquel les fibres nerveuses deviennent protégées par la myéline, substance qui améliore l'efficacité des circuits cérébraux. La myélinisation se poursuivrait tard dans l'adolescence et jusqu'aux premières années de l'âge adulte. ${ }^{4}$ Des connexions neurales plus efficaces au sein du cortex préfrontal seraient nécessaires pour les fonctions cognitives de plus haut niveau telles la planification, l'appréciation des risques et bénéfices et la prise de décisions complexes, régies par des zones préfrontales multiples travaillant de concert.

4. Jusqu'à tard dans l'adolescence, une augmentation de l'efficacité des connections entre le cortex préfrontal et le système limbique. Ce changement serait majeur pour la gestion des émotions, qui est facilitée par une augmentation de la connectivité entre ces régions importantes pour le traitement des informations émotionnelles et le contrôle de soi.

Dans le même exposé, Steinberg poursuit en indiquant que, quant aux changements fonctionnels, des réactions cérébrales concordant avec les changements structurels observés auraient été constatées, soit :

1. Un renforcement de l'activité des systèmes impliquant l'autorégulation: durant les épreuves demandant un contrôle de soi, les adultes utiliseraient un plus large réseau des régions du cerveau que les adolescents plutôt que de surcharger un nombre plus limité de régions, permettant un meilleur contrôle de soi (" self-control »).

2. La réaction face à une promesse de récompense: les adolescents y seraient plus sensibles que les enfants ou les adultes, les entraînant à poser des actes plus risqués (comme la conduite sexuelle ou automobile dangereuse ou l'expérimentation de drogues), surtout en présence de leurs pairs.

3. Une augmentation de l'implication simultanée de plusieurs régions du cerveau répondant à des stimuli provocateurs, mais un moins grand " dialogue » entre les régions du cerveau permettant la régulation de la décision rationnelle et celles qui permettent le contrôle des émotions ; ceci expliquerait une moins grande aptitude à la planification, à l'évaluation des risques et bénéfices et au contrôle des impulsions. Cette particularité du cerveau adolescent pourrait enfin être une cause de la diminution de l'influence des pairs à l'arrivée de l'âge adulte : en acquérant de la maturité, le cerveau deviendrait plus apte à freiner les impulsions suscitées par l'influence des pairs.

Steinberg conclut de tous ces changements que les systèmes impliquant les habiletés cognitives de base atteignent un niveau de maturité comparable à celui des adultes à la mi-adolescence, soit vers 15 ans, alors que ceux qui sont actifs dans l'autorégulation et les habiletés cognitives de plus haut niveau n'arrivent pas à maturité avant le début de l'âge adulte. Par conséquent, les adolescents acquerraient une maturité intellectuelle avant d'acquérir une maturité sociale ou émotive. De plus, il ne serait pas possible d'établir avec précision, en fonction d'un âge déterminé, à quel moment la maturité cérébrale est acquise.

Les recherches en neurosciences et autres disciplines au sujet du développement du cerveau des adolescents se poursuivent. À titre d'exemple, mentionnons les travaux de Galvan et Rahdar sur l'influence du stress quotidien vécu par les adolescents sur leurs performances cognitives, associées à leurs corollaires neuraux, qui concluent à une plus grande vulnérabilité du cerveau en développement au stress [10,11]; ceux de Rodrigo sur les bases neurophysiologiques de la perception différente du risque par les adolescents, en regard des conséquences pour soi-même et pour les autres [12] ; ceux de Blakemore, confirmant également les bases neurophysiologiques des

${ }^{4}$ Soit une période se situant entre 18 et 23 ans, selon les études citées par Steinberg. 
différences observées chez les adolescents dans la prise de décision en présence de pairs ou autres contextes émotifs [13]. Nous notons aussi l'hypothèse intéressante de Wilhems et Reyna qui postulent que l'intuition et la compréhension de l'essentiel (" gist ») des facteurs de décision chez les adultes offrirait un caractère protecteur dans la prise de décision, laissant moins de place aux émotions que chez les adolescents [14].

\section{Enjeux éthiques}

\section{Portée des données provenant des neurosciences}

Pouvons-nous attribuer à ces données une portée suffisante pour remettre en cause la capacité de jugement de l'adolescent en matière de prises de décisions médicales? Plusieurs auteurs, en majorité américains, se sont penchés sur la question depuis quelques années [9,14-23]. Les positions adoptées peuvent se distinguer par leur approche libérale, favorisant l'autonomie de l'adolescent, ou paternaliste, favorisant le retour de l'autorité parentale et ce, de façon similaire aux tendances observées en regard des données scientifiques provenant des recherches en psychologie [24].

Certains auteurs ont argué que ces données ne pouvaient, à elles seules, être déterminantes dans l'évaluation de la capacité de jugement des adolescents: provenant d'expériences faites en laboratoire, elles faisaient abstraction d'autres facteurs, comme le contexte socio-économique, pouvant influencer la prise de décision dans les cas particuliers. On devait donc résister à la tentation d'établir une relation causale entre les comportements neurophysiologiques et les observations statistiques, surtout si elles pouvaient mener à une atteinte aux droits des adolescents comme, par exemple, une hausse de l'âge requis pour obtenir un permis de conduire [19,21]. Pour d'autres, c'est l'accumulation de données et leur convergence avec celles, déjà connues, provenant de la psychologie comportementale, qui permettrait d'en tenir compte dans le traitement réservé aux adolescents $[9,18,20,23,25]$.

Dans le domaine du consentement aux soins médicaux, les points de vue diffèrent toutefois quant à manière de les intégrer aux pratiques: certains ont privilégié une approche individuelle $[9,20]$ et d'autres une approche collective [14,15,17,22,23]. Selon les résultats rapportés par Steinberg et retenus par les auteurs dans leur analyse, les changements structurels et fonctionnels observés par les chercheurs et ce, pendant une période beaucoup plus longue que celle qu'on avait observée auparavant, pourraient ainsi avoir une incidence sur la capacité de l'adolescent d'exercer son jugement. Placé dans les mêmes circonstances, l'adolescent n'exercerait pas son jugement de la même façon qu'un adulte, surtout sous l'influence du stress ou des pairs, en raison de l'immaturité des mécanismes cérébraux de régulation de la décision rationnelle et du contrôle des émotions.

Pour Steinberg [9], les différences observées entre le cerveau des adolescents et celui des adultes devraient servir à guider les soignants dans le cadre de la relation de soins individuelle, sans toutefois remettre en cause la doctrine du mineur mature. Le médecin devrait donc faire en sorte de créer un contexte favorable, permettant la délibération, soit exempt de l'influence des pairs et d'émotions, et conseiller l'adolescent de façon adaptée à son développement, lui permettant d'évaluer correctement les risques et bénéfices à court et à long terme de la décision. Dans ce contexte, l'adolescent d'environ 15 ans serait en mesure, en raison d'habiletés cognitives suffisantes, de prendre des décisions éclairées en contexte médical.

Toujours dans le cadre de la relation de soins individuelle, Diekema [20], quant à lui, estime que ces données devraient mener à un élargissement du devoir de bienfaisance des soignants à l'égard des adolescents. Plus particulièrement en situation de soins critiques, les conséquences néfastes prévisibles devraient les prévenir contre l'attribution d'un trop grand pouvoir décisionnel aux adolescents. Selon lui, les soignants devraient prendre acte de l'immaturité cérébrale des adolescents 
et n'accepter, en aucun cas, le refus d'une intervention vitale ayant des chances de succès raisonnables.

Pour d'autres, le fait que les mécanismes qui permettent le contrôle des émotions dans la prise de décision et l'évaluation des conséquences à court et long terme arrivent à maturité après même l'âge officiel de la majorité est déterminant, peu importe le contexte décisionnel. La propension à une plus grande prise de risques compromettrait également la capacité décisionnelle de l'adolescent. Ces auteurs prônent le retour juridique de l'autorité parentale stricte pour tous les mineurs. D'une part, l'immaturité cérébrale devrait entraîner une immaturité présumée de tous les adolescents en ce qui concerne les prises de décisions médicales, au même titre que les enfants plus jeunes [14,15,17,22]. D'autre part, les données répertoriées relatives aux effets positifs, pour les adolescents, d'un exercice strict de l'autorité parentale et les statistiques sur les comportements sociaux des adolescents ajouteraient à la nécessité de ce changement de paradigme [15,22].

L'approche du «paternalisme collaboratif » défendu par Piker [24] reprise, plus récemment, par Partridge [23], enfin, retient la proposition générale d'une présomption d'immaturité chez le mineur de moins de 18 ans mais avec un exercice de l'autorité parentale qui tiendrait compte de l'opinion de l'enfant selon son niveau de maturité, dans la recherche de son intérêt supérieur. Cette approche amène aussi l'idée que les parents seraient mieux placés que les médecins ou les tribunaux pour guider et connaître l'intérêt supérieur de leur enfant. Ainsi, les adolescents devraient être vus comme des "apprentis preneurs de décisions" en contexte médical plutôt que comme des personnes possédant une faculté de jugement similaire à celle des adultes.

Nous pouvons constater que, bien que partielles, les données provenant des neurosciences sont suffisamment liées aux éléments fondamentaux de la prise de décision pour que l'on s'y intéresse dans le cadre de la réflexion éthique entourant le pouvoir décisionnel de l'adolescent dans un contexte de soins médicaux. En premier lieu, elles touchent à la capacité factuelle de fournir un consentement éclairé, essentielle à une décision pleinement autonome, qui renvoie à la compréhension et l'appréciation de la nature de la maladie et du traitement (ou de son absence) et de leurs conséquences à court et à long terme. Selon Appelbaum [26], ceci requiert des habiletés de communication, de compréhension, d'évaluation et de raisonnement logique permettant non seulement de comprendre les informations reçues, mais aussi de les personnaliser afin de pouvoir expliquer, de façon rationnelle, les raisons de la décision. Ensuite, elles soulignent l'influence des facteurs contextuels sur l'exercice du jugement. On sait que ceux-ci, dans le cadre de la relation de soins, peuvent impliquer émotions, prises de risques, pressions ou pratiques coercitives de la part de tiers, ou encore éléments de satisfaction immédiate, auxquels les adolescents réagissent de façon particulière.

Dans une perspective de protection de l'intérêt supérieur de l'enfant, il nous apparaît qu'au-delà de considérations idéologiques ou sociales, une approche prudentielle devrait nous mener à tenter de réconcilier ces données scientifiques avec notre régime législatif, dans la mesure où celui-ci constitue une exception à la règle générale de protection des mineurs et que des conséquences néfastes pour les adolescents pourraient découler d'une évaluation ou d'une compréhension erronée de leur capacité décisionnelle réelle. À cet égard, il est intéressant de noter les représentations faites en ce sens par l'Association américaine de psychologie devant certains tribunaux en matière criminelle, et la prise en compte de ces données par ceux-ci [27].

\section{Autonomie et capacité décisionnelle de l'adolescent de 14 ans et plus}

La place accordée à l'autonomie, dans la prise de décision de l'adolescent en matière de soins médicaux, s'accorde-t-elle avec ce que nous savons maintenant du développement de son cerveau et coïncide-t-elle réellement avec la protection de son intérêt supérieur? Tout d'abord, nous rappelons que, bien que correspondant à l'acquisition de la "maturité cognitive " selon les travaux en psychologie développementale de Piaget [20], le critère d'âge de 14 ans prévu dans le Code civil y a 
été intégré de manière plus ou moins arbitraire il y a plus de trente ans, suite à un compromis politique [6]. Or, tel que le rappelait la juge Abella dans l'affaire A.C. après avoir fait la recension des publications d'universitaires sur la question, la capacité cognitive de prendre des décisions n'est qu'un de la « myriade de facteurs subtils susceptibles d'influer sur la capacité des adolescents de décider, de façon mature, stable et indépendante, de leur traitement médical » [7, par. 70-1].

À première vue, donc, l'imposition d'un critère rigide fondé sur l'âge de l'adolescent est contredite à la fois par les données scientifiques et l'analyse du Cour Suprême du Canada. Cette situation nous conduit à plusieurs incohérences et difficultés d'application; nous en mentionnerons deux, à titre d'observations préliminaires.

En premier lieu, il nous faut constater le paradoxe de la différence de traitement entre le droit de consentir et le droit de refuser les soins. Nous avons donc, en droit québécois, à la fois un critère établissant que les adolescents de 14 ans et plus, en tant que groupe, sont présumés suffisamment mûrs pour consentir seuls aux soins, mais pas toujours pour les refuser, puisque le tribunal peut être appelé à autoriser un traitement refusé. Dans les faits, un désaccord entre le soignant et l'adolescent peut donc se traduire par une judiciarisation du conflit [1, art. 16, al. 2], alors que pour le mineur de moins de 14 ans c'est le désaccord entre le soignant et les parents qui pourra être soumis à l'examen du tribunal [1, art. 16, al.1]. Ensuite, le soignant se retrouve en quelque sorte juge et partie dans les cas particuliers : en effet, il doit à la fois évaluer l'aptitude de l'adolescent à consentir, la qualité du consentement donné et son meilleur intérêt médical. Ainsi doit-il fournir une information complète et objective à ce dernier, s'assurer de la compréhension de l'information, vérifier que ses facultés mentales ne soient pas altérées, entre autres par la maladie, et l'absence de coercition [5] en plus de déterminer si la décision de l'adolescent est conforme à son meilleur intérêt médical.

Réserver l'utilisation des découvertes neuroscientifiques aux cas particuliers, selon l'approche proposée par Steinberg [9], nous semble poser des difficultés d'application pratique. En effet, ces connaissances pourraient entraîner une confusion supplémentaire entre l'évaluation de l'aptitude de l'adolescent à consentir à un traitement, sa capacité décisionnelle réelle et celle de son meilleur intérêt. Comment déterminer si la décision de l'adolescent est le résultat d'une délibération mûre et rationnelle, exempte de pressions de la part de tiers et correspondant à la fois à une maturité cérébrale et à un conseil adapté à l'acquisition progressive des habiletés nécessaires à l'évaluation des risques et bénéfices à court et à long terme de sa décision? Dans quelles circonstances pourra-ton déterminer que son cerveau a atteint une maturité suffisante pour en conclure que, même pour des raisons contraires à son intérêt médical, sa décision devrait être respectée?

La variabilité des pratiques médicales nous semble être un obstacle suffisamment important pour qu'en pratique, l'évaluation de la décision de l'adolescent puisse toujours se faire en tenant compte de sa réalité neurophysiologique. Si l'on peut penser que les hôpitaux pédiatriques ont développé une expertise en ce domaine, nous pouvons douter d'une telle expertise de la part d'autres praticiens généralistes. Plus important, le Code civil du Québec ne dirige pas le soignant vers une telle adaptation de sa pratique. À cet égard, suivant la rencontre, chapeautée par l'Organisation mondiale de la Santé, d'un groupe de 20 experts en juin 2014, Michaud et collègues [28] ont fait état de la diversité des normes internationales et des pratiques cliniques en matière de détermination de la capacité décisionnelle des adolescents en contexte de soins médicaux. Le groupe propose une procédure délibérative visant à diriger les soignants vers une pratique où les facteurs contextuels et psychosociaux joueraient un rôle prépondérant, et soulignent les défis que représente la détermination de la capacité décisionnelle des adolescents dans les cas particuliers.

De plus, l'on peut craindre que ces facteurs ne soient considérés que dans les cas de refus de traitement, lorsqu'il y a désaccord entre l'adolescent et le médecin. Dit autrement, l'on peut penser qu'il est possible que le soignant se questionne plus aisément quant à la capacité décisionnelle réelle de l'adolescent lorsqu'il fait face à un refus de traitement qu'il estime manifestement déraisonnable 
que lorsque l'adolescent adhère au traitement proposé $[6,29]$. Nous pouvons également nous questionner quant à l'impact que la possibilité d'un recours au tribunal, prévu en cas d'un tel désaccord entre le médecin et son patient, peut avoir sur la relation de confiance et sur le processus décisionnel [29]. Enfin, l'approche de Steinberg laisse entendre qu'il serait possible, pour le médecin, de créer un contexte de prise de décision idéal, adapté à l'immaturité émotive et sociale du patient. Cette idée, plus amplement développée par Michaud et collègues [28], nous semble devoir être explorée davantage pour discuter de la distinction entre les éléments contextuels qui sont du ressort du médecin, comme la façon de favoriser la participation de l'adolescent à la prise de décision, et les facteurs externes, comme le stress ou l'influence des pairs.

Le réexamen du statut spécial accordé aux adolescents en tant que groupe nous paraît plus prometteur. D'une part, outre la contradiction entre les connaissances scientifiques actuelles et l'octroi juridique d'un pouvoir décisionnel en fonction de l'âge, la différence de régime selon qu'on parle d'un refus ou d'un consentement peut être source de confusion quant à l'essence même du concept d'autonomie. Également, le critère d'âge de 14 ans, laissant entendre une présomption de maturité, ne tient pas compte de la nature ou de la gravité de la maladie, ni de la simplicité ou de la complexité de la prise de décision. La reconnaissance de l'immaturité émotive et sociale des adolescents et de ses conséquences sur la prise de décision pourrait mener à une clarification des éléments d'ambivalence de ce régime particulier.

Nous tendons à favoriser l'approche du " paternalisme collaboratif » de Piker [24], mais avec certaines réserves. Tout d'abord, si une limitation au pouvoir décisionnel de l'adolescent peut s'envisager dans la perspective du bénéfice que pourrait représenter la présence parentale dans le processus décisionnel, il faut rappeler que cette présence doit se concevoir, dans la perspective de l'intérêt supérieur de l'enfant, comme une responsabilité de protection [30]. De nouveaux mécanismes de protection pourraient donc devoir être mis en place afin de prévoir les situations où l'exclusion de la présence parentale serait rendue nécessaire pour des raisons d'accès aux services de santé, de négligence ou d'abus dans des cas particuliers. La transmission des informations à l'adolescent, la prise en compte de son avis [31] ${ }^{5}$ et, dans la mesure du possible, l'obtention de son consentement, nous semblent également essentielles à la protection de son intérêt supérieur et tiennent compte du caractère progressif de l'acquisition de sa maturité cérébrale. Ceci s'accorde avec les principes contenus à la Convention relative aux droits de l'enfant [32] et, également avec le droit de l'enfant à la protection, à la sécurité et à l'attention que ses parents peuvent lui donner, prévu dans le Code civil du Québec (art. 32).

Cela étant, nous sommes d'avis qu'un rétablissement juridique de la relation tripartite entre soignant, parents et enfant et, surtout, du partage des informations avec les parents pourrait faciliter l'examen, par le soignant, de plusieurs facteurs contextuels pouvant être déterminants dans la prise de décision tels que la protection offerte (ou non) par le cadre familial, les questions émotives, la complexité de la décision, l'expérience de la pathologie de l'enfant et les conséquences de la décision sur le suivi thérapeutique. Cette approche est favorisée par les auteurs dans une perspective de prévention des conflits et de prise de décision partagée [28,30,31]. Nous ajoutons qu'elle réconcilie la responsabilité parentale avec une perspective globale des soins pédiatriques non seulement médicale, mais aussi sociale et émotionnelle.

\section{Conclusions}

Nous avons vu que les découvertes des neurosciences sur le développement du cerveau des adolescents questionnent l'habileté de l'adolescent à exercer son jugement en matière de soins médicaux. Ainsi, la capacité décisionnelle des adolescents : 1) varie selon l'adolescent et le contexte de la prise de décision, incluant l'influence des tiers et la complexité de la décision à prendre, 2) si on

\footnotetext{
${ }^{5}$ Dans une étude empirique faite en France auprès des équipes de soins, André et collègues ont constaté une meilleure adhésion au traitement lorsque l'enfant était impliqué dans le processus décisionnel.
} 
la relie à la " maturité cérébrale », est fonction d'un processus continu qui pourrait s'étendre sur une longue période allant jusqu'à la mi-vingtaine et 3) pourrait se distinguer de façon importante de celle des adultes en ce qui concerne la prise de risques, la gestion des émotions et l'appréciation des conséquences à court et à long terme d'une décision.

Au Québec, l'octroi d'un pouvoir décisionnel à l'adolescent de 14 ans et plus cohabite avec la doctrine du « mineur mature » qui s'applique ailleurs au Canada, ce qui nous semble être une première source de confusion, tant pour les soignants, qui doivent se référer à la fois au Code civil du Québec et à des guides de pratique qui peuvent être contradictoires en la matière [4], ${ }^{6}$ que pour les parents et les adolescents. La valeur différente accordée au consentement et au refus de l'adolescent, du point de vue de l'autonomie décisionnelle factuellement vérifiable, nous paraît en être une deuxième. Dans les faits, nous ne connaissons pas la façon dont le consentement aux soins de l'adolescent de 14 ans et plus est géré au Québec par les différents professionnels de la santé: comment les informations sontelles données, à qui, comment les parents sont-ils intégrés ou exclus du processus de décision et quels facteurs sont considérés par toutes les parties? La limite de 14 ans est-elle respectée de façon stricte par tous les professionnels de la santé? Des données empiriques sur les perceptions des participants à la relation de soins et des pratiques cliniques québécoises pourraient venir nourrir la réflexion. Carnevale et collègues [33], qui se penchent sur l'éthique de l'enfance, soulignent la nécessité de développer la recherche, notamment dans les domaines de la médecine et du droit, afin d'étudier les relations entre les concepts de meilleur intérêt de l'enfant, autonomie, autorité parentale et reconnaissance de l'enfant comme agent moral.

Nous ne pouvons enfin passer sous silence l'article 5 de la nouvelle Loi concernant les soins de fin de vie [34], qui prévoit le droit du mineur de 14 ans et plus de refuser ou de demander le retrait d'un traitement qui a pour but de le maintenir en vie et ce, " dans la mesure prévue par le Code civil ». Nous nous interrogeons quant au poids qui sera accordé par les soignants, ou par les tribunaux, à la volonté de l'adolescent lorsque les conséquences prévisibles de la décision sont le décès. Nous nous demandons également dans quelle mesure, dans la foulée de l'arrêt Carter relatif au suicide médicalement assisté [35], ${ }^{7}$ ceci pourra ouvrir la porte, dans le futur, à l'extension de ce droit à celui de demander l'aide médicale à mourir (qui est définie comme étant un soin aux termes de cette loi). ${ }^{8}$

Compte tenu de l'écart entre les connaissances actuelles et celles qui prévalaient lors de l'adoption de ce régime particulier, une nouvelle évaluation du bien-fondé des articles 14, al. 2 et 16, al. 2 du Code civil en regard des données provenant des neurosciences, ainsi que des pratiques cliniques, nous paraît souhaitable. Nous suggérons qu'un régime uniformisé tout au long de la minorité (et même plus tard) qui pourrait comporter des exceptions basées sur la gravité de la condition de l'enfant, la durée de sa maladie ou les situations de négligence parentale pourrait être mieux adapté aux connaissances scientifiques actuelles et correspondre à la protection de l'intérêt supérieur de l'adolescent, en plus de favoriser une prise en charge et un suivi thérapeutique optimaux.

\footnotetext{
${ }^{6} \bar{A}$ titre d'exemple, le Document de principes du comité de bioéthique de la Société canadienne de pédiatrie fait état des éléments de maturité et de risque de dommages reliés à l'intervention en précisant que la capacité n'est pas reliée à l'âge ou à la maladie.

${ }^{7}$ L'arrêt Carter, bien que touchant les adultes, mentionne le jugement A.C. lorsqu'il est question du principe du respect de l'autonomie.

${ }^{8}$ Ce droit est réservé aux adultes aux termes de cette loi.
} 


\section{Références}

1. Code civil du Québec, L.Q. 1991, c. 64.

2. Kouri RP. Le mineur et les soins médicaux, A.C. C. Manitoba : de l'autonomie au meilleur intérêt, une limite bien floue. McGill Journal of Law and Health. 2010. 4(1): 65-81.

3. Ferguson L. The End of an Age: Beyond Age Restrictions for Minors' Medical Treatment Decisions (29 octobre 2004). Social Science Electronic Publishing Inc.

4. Harrison $\mathrm{C}$, Société canadienne de pédiatrie (SCP) Comité de bioéthique. Les décisions de traitement au nom des nourrissons, des enfants et des adolescents. Paediatr Child Health 2004;9(2):109-14.

5. Deleury É, Goubau D. Le droit des personnes physiques. 4e éd. Cowansville (Qc): Yvon Blais; 2008.

6. Kouri RP, Philips-Nootens S. Les soins requis par l'état de santé. L'intégrité de la personne et le consentement aux soins. 3e éd. Cowansville: Yvon Blais, une société de Thomson Reuters; 2012.

7. A.C. c. Manitoba (Directeur des services à l'enfant et à la famille). [2009] 2 R.C.S.181.

8. Code de la santé publique, Article L1111-4

9. Steinberg L. Does recent research on adolescent brain development inform the mature minor doctrine? J Med Philos. 2013;38(3):256-67.

10. Galvan A, Rahdar A. The neurobiological effects of stress on adolescent decision making. Neuroscience. 2013;249:223-31.

11. Rahdar A, Galvan A. The cognitive and neurobiological effects of daily stress in adolescents. Neuroimage. 2014;92:267-73.

12. Rodrigo MJ, Padrón I, de Vega M, Ferstl EC. Adolescents' risky decision-making activates neural networks related to social cognition and cognitive control processes. Frontiers in Human Neuroscience. 2014;8:60.

13. Blakemore SJ, Robbins TW. Decision-making in the adolescent brain. Nat Neurosci. 2012;15(9):1184-91.

14. Wilhelms EA, Reyna VF. Fuzzy trace theory and medical decisions by minors: differences in reasoning between adolescents and adults. J Med Philos. 2013;38(3):268-82.

15. Partridge BC. The mature minor: some critical psychological reflections on the empirical bases. J Med Philos. 2013;38(3):283-99.

16. Partridge BC. The Decisional Capacity of the Adolescent: An Introduction to a Critical Reconsideration of the Doctrine of the Mature Minor. Journal of Medicine and Philosophy. 2013;38(3):249-55.

17. Cherry MJ. Ignoring the data and endangering children: why the mature minor standard for medical decision making must be abandoned. J Med Philos. 2013;38(3):315-31.

18. Edwards TM. Capacity and the Adolescent Brain. Psychiatry, Psychology and Law. 2009;16(3):427-34.

19. Males M. Does the Adolescent Brain Make Risk Taking Inevitable?: A Skeptical Appraisal. Journal of Adolescent Research. 2009;24(1):3-20.

20. Diekema DS. Adolescent refusal of lifesaving treatment: are we asking the right questions? Adolesc Med State Art Rev. 2011;22(2):213-28, viii.

21. Johnson SB, Blum RW, Giedd JN. Adolescent maturity and the brain: the promise and pitfalls of neuroscience research in adolescent health policy. J Adolesc Health. 2009;45(3):216-21.

22. Barina R, Bishop JP. Maturing the minor, marginalizing the family: on the social construction of the mature minor. J Med Philos. 2013;38(3):300-14.

23. Partridge B. Adolescent Pediatric Decision-Making: A Critical Reconsideration in the Light of the Data. HEC Forum. 2014;26(4):299-308.

24. Piker A. Balancing liberation and protection: a moderate approach to adolescent health care decision-making. Bioethics. 2011;25(4):202-8.

25. Steinberg L. Should the science of adolescent brain development inform public policy? Am Psychol. 2009;64(8):739-50. 
26. Appelbaum PS. Clinical practice. Assessment of patients' competence to consent to treatment. N Engl J Med. 2007;357(18):1834-40.

27. Steinberg $L$. The influence of neuroscience on US Supreme Court decisions about adolescents' criminal culpability. Nat Rev Neurosci. 2013;14(7):513-8.

28. Michaud P-A, Blum RW, Benaroyo L, Zermatten J, Baltag V. Assessing an Adolescent's Capacity for Autonomous Decision-Making in Clinical Care. Journal of Adolescent Health. 2015;57(4):361-6.

29. Cave E. Goodbye Gillick? Identifying and resolving problems with the concept of child competence. Legal studies. 2014;34(1):103-22.

30. Pinnock R, Crosthwaite J. When parents refuse consent to treatment for children and young persons. J Paediatr Child Health. 2005;41(7):369-73.

31. André N, Gaudart J, Bernard JL, Chabrol B. Quelle place pour l'enfant dans la prise de décision en pédiatrie ? Archives de Pédiatrie. 2005;12(7):1068-74.

32. Assemblée Générale des Nations-Unies. Convention relative aux droits de l'enfant. NationsUnies: Recueil des Traités, Vol 1577,1989.

33. Carnevale FA, Campbell A, Collin-Vézina D, Macdonald ME. Interdisciplinary Studies of Childhood Ethics: Developing a New Field of Inquiry. Children \& Society. 2015;29(6):511-23.

34. Loi concernant les soins de fin de vie, LQ 2014, c. 2.

35. Carter c. Canada (Procureur général) [2015] 1 RCS 331.

36. Silber TJ. Adolescent brain development and the mature minor doctrine. Adolesc Med State Art Rev. 2011;22(2):207-12, viii.

37. Blakemore S-J. Imaging brain development: The adolescent brain. Neurolmage. 2012;61(2):397-406.

38. Verma S, Silberfeld M. Approaches to capacity and competency: The Canadian view. International Journal of Law and Psychiatry. 1997;20(1):35-46.

39. Partridge BC. Adolescent psychological development, parenting styles, and pediatric decision making. The Journal of medicine and philosophy. 2010;35(5):518.

40. Bailly D. Problèmes liés au consentement chez l'enfant et l'adolescent. Archives de Pédiatrie. 2010;17, Supplement 1(0):S7-S15.

41. Smith AR, Chein J, Steinberg L. Impact of socio-emotional context, brain development, and pubertal maturation on adolescent risk-taking. Horm Behav. 2013;64(2):323-32.

42. Lelièvre N. Adolescent et soins, vers une autonomie de la volonté ? Douleurs : Evaluation Diagnostic - Traitement. 2005;6(4, Part 1):247-53. 